Short Communication

\title{
What is in a name? \\ The development of cross-cultural differences in referential intuitions
}

\author{
Jincai $\mathrm{Li}^{\mathrm{a}}{ }^{\mathrm{b}, *}$, Longgen $\mathrm{Liu}^{\mathrm{a}}$, Elizabeth Chalmers ${ }^{\mathrm{b}}$, Jesse Snedeker ${ }^{\mathrm{b}}$ \\ ${ }^{a}$ School of Foreign Languages, Shanghai Jiao Tong University, No. 800 Dongchuan Road, Minhang District, Shanghai 200240, China \\ b Department of Psychology, Harvard University, 33 Kirkland St, Cambridge, MA 02138, USA
}

\section{A R T I C L E I N F O}

\section{Keywords:}

Proper names

Descriptivist theory

Causal-historical theory

Referential intuitions

Cross-cultural differences

Development

\begin{abstract}
A B S T R A C T
Past work has shown systematic differences between Easterners' and Westerners' intuitions about the reference of proper names. Understanding when these differences emerge in development will help us understand their origins. In the present study, we investigate the referential intuitions of English- and Chinese-speaking children and adults in the U.S. and China. Using a truth-value judgment task modeled on Kripke's classic Gödel case, we find that the cross-cultural differences are already in place at age seven. Thus, these differences cannot be attributed to later education or enculturation. Instead, they must stem from differences that are present in early childhood. We consider alternate theories of reference that are compatible with these findings and discuss the possibility that the cross-cultural differences reflect differences in perspective-taking strategies.
\end{abstract}

\section{Introduction}

At birth, we are all given a name, which usually follows us through life. When people use your name, they typically refer to you. But what is the mental link that ties a name to a person and allows it to refer?

Two well-known proposals in the philosophical literature seek to answer this question. The descriptive view, defended by Frege (1892/ 1948), Russell (1905) and Searle (1958) among others, holds that a name gets its referent through definite descriptions. When competent speakers use a name, they refer to whoever uniquely satisfies the description associated with that name. For instance, the name "Barack Obama" refers to Barack Obama because he is the person best fitting the definite description "the 44th President of the United States". On this account, names refer indirectly, mediated by definite descriptions in the speaker's mind. The second proposal, Kripke's causal-historical view, contends that a name refers to a person because it was linked to her in the initial act of naming and this link is then passed down through a community of speakers. Kripke argues that proper names are rigid designators; they continue to refer to the entity initially given the name, even when that individual turns out to have none of the properties we associate with that name (Kripke, 1972/1981). On this account, names refer directly without the mediation of definite descriptions. ${ }^{1}$

Kripke supported his proposal with a famous thought experiment. He noted the only thing most people have heard about mathematician
Kurt Gödel is that he was the person who proved the incompleteness of arithmetic, so this is the only possible definite description that they could associate with Gödel. Now, imagine that Gödel actually stole the theorem from someone named Schmidt, who did all the work. According to descriptivism, when people use the name "Gödel", they really refer to Schmidt, who is the unique person satisfying the definite description they have. Kripke's intuitions, in contrast, tell him that speakers use the name "Gödel" to refer to whoever was given that name initially (Kripke, 1972: 83-4).

Kripke's intuitions about the Gödel case were widely shared among philosophers, and thus the descriptive theory lost favor. Machery, Mallon, Nichols, and Stich (2004), however, questioned the universality of Kripkean intuitions. They presented stories modeled on the Gödel case to undergraduates in the U.S. and China and discovered considerable variation in people's semantic intuitions. While some people have causal-historical intuitions, others have descriptivist intuitions. Additionally, these intuitions vary systematically by culture: while American participants generally endorsed the causal-historical view, Chinese participants mostly endorsed descriptivism. Subsequent experiments, varying the stimuli and the populations, have replicated this pattern (e.g., Beebe \& Undercoffer, 2015; Beebe \& Undercoffer, 2016; Machery, Sytsma, \& Deutsch, 2015; Machery et al., 2010; Sytsma, Livengood, Sato, \& Oguchi, 2015).

To date, however, researchers have not investigated the specific

\footnotetext{
* Corresponding author at: School of Foreign Languages, Shanghai Jiao Tong University, No. 800 Dongchuan Road, Minhang District, Shanghai 200240, China.

E-mail address: caralee@sjtu.edu.cn (J. Li).

${ }^{1}$ There are other theories of reference in the philosophical literature, some of which we consider in the General Discussion. We focus on these two because they are addressed directly by Kripke's thought experiment (1972) and the subsequent work in experimental philosophy.
} 
causes of the cross-cultural variation. Determining when in development these differences appear is a critical first step in doing so, as it will help us to identify potential causes. For example, differences emerging in college years would suggest formal education in science or philosophy plays a role. In contrast, if the difference is present by age seven, it is likely to arise from cross-cultural differences in early social interaction and communication. Exploring the developmental trajectory of the differences could also inform us about the initial basis of reference. We hypothesize that there are four possible developmental pathways:

I. Initial Descriptivism: Children begin with a descriptivist theory of reference regardless of culture. English-speaking children move towards the causal-historical view during development. This suggests that referential links are based primarily on descriptions, and causal-historical intuitions result from later education and socialization.

II. Initial Causal-Historical: Children begin with a causal-historical theory of reference regardless of culture. Chinese-speaking children shift towards descriptivism as they grow. This pattern suggests that causal-historical chain serves as the initial basis for reference, with descriptivist intuitions arising from subsequent socialization and education.

III. Early Differentiation: Children in both groups possess a culturally specific theory of reference at a young age, acting like adults from their culture and exhibiting systematic differences in their referential intuitions. This indicates that divergence takes place in early childhood.

IV. Initial Ambivalence: Children in neither culture have developed a specific strategy for fixing the reference of names, and are equally likely to rely on descriptive intuitions and causal-historical intuitions. This pattern could arise because individual children hold both theories or because different children utilize different theories.

Testing these hypotheses in young children calls for tools that do not require counterfactual reasoning or meta-linguistic judgments as the standard probes do (see Machery et al., 2004: B6-7). The truth-value judgment task (Crain \& McKee, 1985), adopted widely in developmental psychology, can be used with children as young as three. ${ }^{2}$ If there are genuine cross-cultural differences in intuitive judgments about reference, then we should see a similar pattern when asking questions about truth, since judgments about the truth of a name-containing statement depend on what the referent of the name is (see Machery, Olivola, \& de Blanc, 2009 for related arguments).

\section{Experiment}

\subsection{Participants}

Forty English-speaking children in the U.S. (age: 6;6-8;4; mean 7;4; 22 girls) and thirty Mandarin-speaking children in China (age: 6;6-8;3; mean 7;2; 17 girls) participated. There was no reliable age difference between these groups $(\mathrm{t}(68)=-1.15, \mathrm{p}=0.25)$. Additionally, fortyseven adult English speakers in the U.S. (mean age $=21.4$; 32 female) and forty-seven adult Mandarin speakers in China (mean age $=20.7$; 32 female) participated.

\subsection{Materials}

The stimuli consisted of two critical stories and three familiarization stories (see Supplementary materials). We constructed stories similar to

\footnotetext{
${ }^{2}$ We are aware of the controversy over the appropriate approach to gauge intuitions about reference in the philosophical and experimental philosophy literature (see Devitt, 2011; Devitt, 2012; Devitt, 2015; Domaneschi, Vignolo, \& Di Paola, 2017; Martí, 2009; Martí, 2012). But due to space limitations, we will not plunge into the debate in this brief article.
}

the original Gödel case about topics that are more appropriate for young children. A simplified version of one critical story is given below:

\section{Super Dog Race}

Long ago, there was a race called the Super Dog Race. Max, Pickles and Blaze participated in the race. Max crossed the finish line first, winning the race, but he got too excited and ran all the way to the North Pole. Pickles crossed the finish line second. He stopped and watched Max run away. The race announcer mistakenly thought that Pickles won the race. He told every newspaper in the world that Pickles won. He also told them that another dog, Blaze, ran very fast despite his short legs. Since then, everyone learned that Pickles won the race. They don't know anything else about Pickles.

Tom and Emily learned at school that Pickles won the Super Dog Race. This is the only thing they know about the dog race and Pickles. They don't know anything about Max. That night, their dad asked: Do you know who won the Super Dog Race?

Tom replied: Blaze was the dog that won the Super Dog Race. Emily said: Pickles was the dog that won the Super Dog Race.

Tom's clearly false statement is a control. Emily's statement is the critical statement that elicits participants' referential intuitions. It is true if the name "Pickles" gets its reference from the definite description in her head ("the dog that won the Super Dog Race"). It is false if reference is based on a causal-historical chain such that "Pickles" necessarily picks out the original bearer of this name, regardless of any associated descriptions. Accordingly, a "Yes" answer is considered a descriptive response, while a "No" answer is considered a causal-historical response.

The familiarization stories are similar, but the reference of the names in Tom and Emily's statements is unambiguous. Both statements are true in one story, both false in another, and one is true and one is false in the third. Thus, including the two control statements in the critical stories, there are eight statements with determinate answers, three that are true and five that are false. We label them as Yes-controls and No-controls respectively.

The stories are accompanied by clipart pictures to engage participants. We randomized the order of the familiarization stories and the critical stories for each adult participant. For child participants, we created two lists with the stories appearing in different orders. The order of the two statements in each story was counterbalanced. A native speaker (J.L.) translated the probes into Chinese for use with Chinesespeaking participants. All names in the translated probes were typical Chinese names.

\subsection{Procedure}

Children sat in front of a screen in the lab and saw the pictures while an experimenter read the stories aloud from a script and recorded their verbal responses. Adult participants accessed the study on Qualtrics through an anonymous survey link. They read each story, with the pictures interspersed, and answered the relevant questions before proceeding to the next. After all five stories, they completed a short demographic questionnaire.

\subsection{Results and analysis}

Table 1 displays the percentage of correct responses to the control questions. While children in both cultures made more errors than adults, no group answered less than $80 \%$ of the questions accurately, indicating that the task was manageable even for the children.

To determine whether there were any cultural differences in the responses to the control statements, a binomial mixed-effects model was constructed using the $\mathrm{R}$ programming language, with culture, age and their interaction as fixed effects and participant and statement as random effects. ${ }^{3}$ We found a main effect of age $(z=6.274, p<0.001)$ but no main effect of culture $(\mathrm{z}=0.031, \mathrm{p}>0.1)$ and no interaction 
Table 1

Percentage of correct responses to all control statements.

\begin{tabular}{llllll}
\hline & \multicolumn{2}{l}{ Children } & & & \multicolumn{2}{l}{ Adults } \\
\cline { 2 - 3 } \cline { 5 - 6 } & American & Chinese & & American & Chinese \\
\hline Yes-Controls & $86 \%$ & $80 \%$ & & $93 \%$ & $91 \%$ \\
No-Controls & $85 \%$ & $80 \%$ & & $95 \%$ & $99 \%$ \\
\hline
\end{tabular}

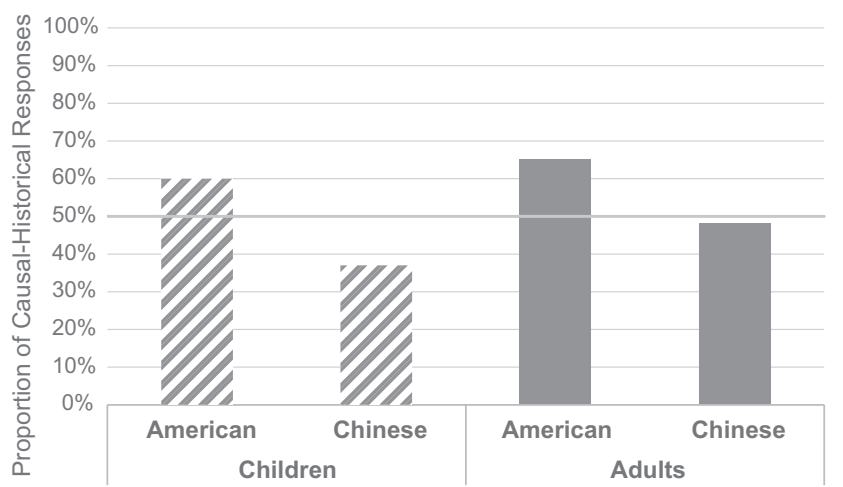

Fig. 1. Proportion of causal-historical response to critical statements.

between age and culture $(\mathrm{z}=1.697, \mathrm{p}>0.05)$. Separate analyses of the responses to Yes-controls and No-controls also found no reliable effects or interactions of culture (all p's $>0.1$ ). Thus the two cultural groups did not differ reliably in their response biases or accuracy.

For the critical statements, "No" responses (coded as 1) were our dependent variable. As Fig. 1 shows, while children and adults in the U.S. responded "no" the majority of the time $(60 \%$ and $65 \%$ respectively), children and adults in China did so less often (37\% and $48 \%$ respectively). To assess this pattern, a binomial mixed-effects model, with the same specification as the one above, was created. We found a main effect of culture $(\mathrm{z}=-2.841, \mathrm{p}<0.01)$ but no effect of age $(\mathrm{z}=1.243, \mathrm{p}>0.1)$ or interaction between culture and age $(\mathrm{z}=0.448, \mathrm{p}>0.1)$. In short, Americans in both age groups are more likely to respond to the critical statements in a manner consistent the causal-historical view of reference than the Chinese are.

\section{General discussion}

This study produced two clear findings. First, we replicated the cross-culture difference observed by Machery et al. (2004) using a truth-value judgment task. Determining the truth of a statement, in this context, requires participants to determine the referent of the name occurring in it. If we take the name to refer to the entity who was originally given the name (consistent with the causal-historical view), then the statement is false. If we take the name to refer to the person who actually matches the description in the speaker's head (consistent with the descriptive hypothesis), then the statement is true. While our method differs from Machery and colleagues', the pattern of findings is parallel. When fixing the reference of proper names in Gödel-style probes, American adults are more likely to respond like causal theorists, whereas Chinese adults give more responses consistent with the descriptive theory.

Second, and most critically, we observed the same pattern in 7-yearold children. To the best of our knowledge, this is the first study to explore the development of cross-cultural variation in referential intuitions. These findings support the early differentiation hypothesis by

\footnotetext{
${ }^{3}$ Correct responses were coded as 1 . The model specification was: Controls.lmer $=$ glmer $\quad($ Correctness $\sim$ Culture *Age $+(1 \mid$ Participant $)+(1 \mid$ Statement $)$, data $=$ Controls, family $=$ binomial $)$.
}

demonstrating that children already have culturally specific referential intuitions by age seven. We found no evidence for either the initial descriptivism hypothesis (as American children already favored the causal-historical view) or the initial causal-historical hypothesis (as Chinese-speaking children already favored the descriptive view). How early this differentiation emerges is still an open question. It is possible that one theory is present in all cultures at a younger age, or that children are ambivalent earlier in development.

These findings raise two questions: what causes the cross-cultural differences and how does the presence of variation constrain our theories of reference? The distinct cultural patterns at age seven suggest that formal education in science or philosophy and late socialization play little role. Instead, the variation must arise from differences in experience prior to this age, such as early socialization or language learning. We consider three possibilities below.

One possibility is that our cross-cultural findings result from different interpretations of the specific events chosen for the critical stories. This is because actions like winning a race or discovering an artifact could be socially or externally defined, making them somewhat ambiguous. For instance, maybe for the Chinese, who belong to a more collectivist culture, the "winner of the race" is the person declared the winner by the judge rather than the person who crossed the finish line first. Likewise, they may consider the "discoverer of Claymen" to be the person who first shared that knowledge with others rather than the person who initially uncovered the clay figures. If this is the case, it could be that what differs across cultures is the construal of the predicates, rather than the reference of the names themselves. However, in a follow-up study using four new stories that do not involve actions that can be socially defined (e.g., building a hospital, writing a book, baking a cake, and drawing pictures), we replicated the cultural effect robustly. Adult participants from China provided causal-historical responses $34 \%$ of the time, while American participants responded causally $90 \%$ of the time. We therefore believe it is unlikely the cross-cultural patterns stem from divergent understanding of the actions involved in the vignettes.

Another possibility is that these findings could reflect differences in norms of politeness in Chinese and American culture. Since the Chinese are generally harmony-oriented and tend to avoid contradicting others (Intachakra, 2012; Nisbett, 2003:45; 173-190), they might be less likely than Americans to reject another's statements in our truth-value judgment task. Our analysis of responses to control statements rules out a strong version of this hypothesis: there were no differences between the two cultural groups in their accuracy for either the Yes- or Nocontrols. Thus the Chinese subjects readily contradicted an imaginary speaker when she was unambiguously wrong. This leaves open the possibility that politeness norms play a subtler role. For example, if participants have access to both interpretations of the ambiguous names, politeness considerations might affect which of the two they favor.

The third hypothesis is that different perspective-taking strategies induce cross-cultural variations in people's referential intuitions. Research in cultural psychology has found that Chinese adults and children are better perspective takers than their American counterparts (Luk, Xiao, \& Cheung, 2012; Sabbagh, Xu, Carlson, Moses, \& Lee, 2006; Wu, Barr, Gann, \& Keysar, 2013; Wu \& Keysar, 2007). Our critical stories, and Gödel-style probes more generally, depend on an asymmetry in the knowledge state of the embedded speaker (e.g., Emily) and the participant (Sytsma \& Livengood, 2011). Emily believes that Pickles is the winner of the race but knows nothing else. The participant knows that Emily believes this, knows that it is incorrect, and, by necessity, knows a bit more about Pickles (e.g., that there was a dog dubbed Pickles who did not win the race). Consequently, how we fix the reference of the name "Pickles" may depend on whose perspective we access most readily. If Emily's perspective is immediately available to us, we might gravitate toward the descriptivist intuition and judge the statement true. In contrast, if our own perspective is more salient, we might privilege causal-historical information and reject the statement. 
On this proposal, Chinese speakers have descriptivist intuitions more often because they are more likely to take the embedded speaker's perspective.

It is critical to note that we found considerable variation within each of the groups we studied: some Chinese speakers provided only causalhistorical responses, some English speakers provided only descriptivist responses, and many participants gave responses of both kinds. Thus it would be unwise to characterize any particular cultural group as being "pure descriptivists" or "pure causal theorists" (see Genone \& Lombrozo, 2012; Nichols, Pinillos, \& Mallon, 2016). Instead, it seems that people in both groups have access to multiple strategies for fixing reference and shift between them, perhaps based on factors like politeness or perspective taking.

What are those strategies for reference-fixing? At the beginning of the paper, we introduced the two possibilities that motivated the Gödel story and the subsequent research in experimental philosophy: (1) reference is fixed by a description, that is either in the head of the speaker or shared by the linguistic community (resulting in acceptance of the critical statement) or (2) reference is fixed by a causal-historical link that is established at birth and cannot be broken (resulting in rejection). There are, however, alternate forms of descriptivism and the causalhistorical theory which complicate the interpretation of our findings, and all the previous studies relying on Gödel-style probes. For example, it is possible that people always fix reference on the basis of a definite description but that the locus and content of that description varies. Some people may rely on the descriptions available to the speaker and her linguistic community (speaker-relative descriptivism) while others rely on the description that would be available to an omniscient, outside observer, such as the narrator or the participant in these studies (assessor-relative descriptivism). ${ }^{4}$ On this account, our data is evidence for a cross-cultural difference in the rate of speaker-relative and assessor-relative descriptivism that emerges by seven. There are also alternative versions of the causal theory that can accommodate the observed variability. For example, perhaps many Americans (and a few Chinese) prefer to fix reference based on a causal-historical chain stretching between an initial naming event and contemporary use of this name (as Kripke proposes). But perhaps for most Chinese (plus a few Americans), the key to reference fixing is what Evans' (1973) called the "dominant source of the causal origin" of the information associated with a name, which in our probes is the false history that had been passed down over generations in Emily's linguistic community. According to this theory, the cross-cultural differences is about which causal links are deemed most relevant.

In sum, previously observed cross-cultural patterns in referential intuitions hold up in a different and arguably more naturalistic truthvalue judgment task. These cross-cultural differences are fully in place by seven years of age. Thus, whatever triggered them must be present in early childhood. The non-categorical response patterns in both cultural groups suggest that the referential mechanism of proper names might be fluid in the sense that it varies across individuals within a culture and perhaps within an individual over time. By exploring this fluidity, we may better understand the cognitive mechanisms underlying referential intuitions.

\section{Acknowledgements}

This work was supported by funding from the China Scholarship Council, the National Natural Science Foundation of China (31471075), as well as the International exchange program between National
Natural Science Foundation of China and Royal Society of the United Kingdom (31611130183). We are grateful to members of the Snedeker Lab and the Laboratory for Developmental Studies at Harvard University for helpful discussion and comments. We also thank the children and adults who participated in this study.

\section{Appendix A. Supplementary material}

Supplementary data associated with this article can be found, in the online version, at http://dx.doi.org/10.1016/j.cognition.2017.10.022.

\section{References}

Beebe, J. R., \& Undercoffer, R. J. (2015). Moral valence and semantic intuitions. Erkenntnis, 80(2), 445-466.

Beebe, J. R., \& Undercoffer, R. J. (2016). Individual and cross-cultural differences in semantic intuitions: New experimental findings. Journal of Cognition and Culture, 16(16), 322-357.

Crain, S., \& McKee, C. (1985). Acquisition of structural restrictions on anaphora Proceedings of the north eastern linguistic society. Vol. 16. Proceedings of the north eastern linguistic society (pp. 94-110). Amherst MA: GLSA.

Devitt, M. (2011). Experimental semantics. Philosophy and Phenomenological Research, LXXXII, 418-435.

Devitt, M. (2012). Whither experimental semantics? Theoria, 73, 5-36.

Devitt, M. (2015). Testing theories of reference. In J. Haukioja (Ed.). Advances in experimental philosophy of language (pp. 31-63). London: Bloomsbury Academic.

Domaneschi, F., Vignolo, M., \& Di Paola, S. (2017). Testing the causal theory of reference. Cognition, 161, 1-9.

Evans, G., \& Altham, J. (1973). The causal theory of names. Proceedings of the Aristotelian Society, Supplementary Volumes, 47, 187-225.

Frege, G. (1892/1948). Sense and reference. The Philosophical Review, 57(3), 209-230.

Genone, J., \& Lombrozo, T. (2012). Concept possession, experimental semantics, and hybrid theories of reference. Philosophical Psychology, 25(5), 717-742.

Intachakra, S. (2012). Politeness motivated by the 'heart' and 'binary rationality' in Thai culture. Journal of Pragmatics, 44(5), 619-635.

Kripke, S. A. (1972/1981). Naming and necessity. Malden, MA, USA: Blackwell Publishing.

Luk, K. K., Xiao, W. S., \& Cheung, H. (2012). Cultural effect on perspective taking in Chinese-English bilinguals. Cognition, 124(3), 350-355.

MacFarlane, J. (2014). Assessment sensitivity: Relative truth and its applications. Oxford, UK: Oxford University Press.

Machery, E., Deutsch, M., Mallon, R., Nichols, S., Sytsma, J., \& Stich, S. P. (2010). Semantic intuitions: Reply to Lam. Cognition, 117(3), 361-366.

Machery, E., Mallon, R., Nichols, S., \& Stich, S. P. (2004). Semantics, cross-cultural style. Cognition, 92(3), B1-B12.

Machery, E., Olivola, C., \& de Blanc, M. (2009). Linguistic and metalinguistic intuitions in the philosophy of language. Analysis, 69, 689-694.

Machery, E., Sytsma, J., \& Deutsch, M. (2015). Speaker's reference and cross-cultural semantics. In A. Bianchi (Ed.). On reference (pp. 62-76). Oxford: Oxford University Press.

Martí, G. (2009). Against semantic multi-culturalism. Analysis, 69, 42-48.

Martí, G. (2012). Empirical data and the theory of reference. In W. Kabasenche, (Ed.). Topics in Contemporary Philosophy. Reference \& Referring (pp. 63-82). MIT Press.

Nichols, S., Pinillos, N.Á., \& Mallon, R. (2016). Ambiguous reference. Mind, 125(497), $145-175$.

Nisbett, R. (2003). The geography of thought: How Asians and Westerners think Differently... and why? New York: Free Press.

Russell, B. (1905). On denoting. Mind, 14(56), 479-493.

Sabbagh, M. A., Xu, F., Carlson, S. M., Moses, L. J., \& Lee, K. (2006). The development of executive functioning and theory of mind a comparison of Chinese and US preschoolers. Psychological Science, 17(1), 74-81.

Searle, J. R. (1958). Proper names. Mind, 67(266), 166-173.

Sytsma, J., \& Livengood, J. (2011). A new perspective concerning experiments on semantic intuitions. Australasian Journal of Philosophy, 89(2), 315-332.

Sytsma, J., Livengood, J., Sato, R., \& Oguchi, M. (2015). Reference in the land of the rising sun: A cross-cultural study on the reference of proper names. Review of Philosophy and Psychology, 6(2), 213-230.

Wu, S., Barr, D. J., Gann, T. M., \& Keysar, B. (2013). How culture influences perspective taking: Differences in correction, not integration. Frontiers in Human Neuroscience, 7 , 822.

Wu, S., \& Keysar, B. (2007). The effect of culture on perspective taking. Psychological Science, 18(7), 600-606. 